\title{
Identificación, estudio preliminar y puesta en valor de las canteras históricas de San Ciprián (Lugo)
}

\author{
J. Fernández Suárez ${ }^{(1)}$, E. Álvarez Areces ${ }^{(1,2)}$, J.M. Baltuille Martín ${ }^{(1,2)}$ y J. Martínez-Martínez ${ }^{(1)}$
}

(1) Unidad de Piedra Natural y Patrimonio Monumental. Instituto Geológico y Minero de España. C/ Ríos Rosas, 23.28003 Madrid. j.fernandez@igme.es; e.alvarez@igme.es; jm.baltuille@igme.es; javier.martinez@igme.es

(2) Red CONSTRUROCK (Red de la Piedra Natural y su relación con el Patrimonio Histórico-Monumental y la obra nueva, en el ámbito del Estado español). Ríos Rosas, 23, 28003 Madrid.

\section{RESUMEN}

El objetivo del este trabajo es poner de manifiesto la existencia y características de las canteras históricas de extracción de piedra para la construcción del Patrimonio Arquitectónico de San Ciprián, pequeña localidad del N de Lugo (Galicia), donde, a lo largo de los siglos las rocas han sido utilizadas como materiales naturales de construcción, fijando así una vista armónica entre la roca y el paisaje. La localización de las canteras históricas tiene, además del componente del conocimiento histórico, socio-económico e industrial, sobre la tecnología aplicada para la explotación, el objetivo de permitir definir el estado original del material, así como proporcionar una nueva aportación al catálogo turístico de la población, aprovechando su identificación y descripción como complemento a las rutas culturales y ambientales de la misma.

A partir de este estudio se pone de manifiesto la existencia y las características de los signos de explotación localizados en las canteras históricas asociadas con el Patrimonio Arquitectónico de San Ciprián, si bien en algunos casos han desaparecido parcial o totalmente.

Las áreas localizadas son: Porto de Riba, Figueiras, Área de la Ría y la cantera de Lieiro; no pudiéndose afirmar la inexistencia de otras zonas de extracción actualmente desaparecidas, especialmente en la Zona de Porto de Riba y Figueiras.

Palabras clave: Canteras históricas, Galicia, Patrimonio Arquitectónico, piedra natural.

\section{Identification, preliminary study and valorization of the historical quarries of San Ciprián (Lugo)}

\begin{abstract}
The aim of this paper is showing the existence and characteristics of the historical quarries where the building stones were extracted for the construction of the Architectural Heritage of San Ciprián. It is a small town in the north of Lugo (Galicia, NW of Spain) with a long tradition in the use of the natural stone as building material in its architectural heritage. In addition to the benefits in the historical, socio-economic and industrial knowledge, the location of historical quarries is a fundamental task for the conservation plans, allowing to define the original state of the building stone. Moreover, they provide a new contribution to the regional tourist development, including their location and description in the cultural and environmental touristic routes.

This study reveals the existence and characteristics of the signs of operation located in the historical quarries associated with the Architectural Heritage of San Ciprián, although in some cases this quarries have disappeared partially or totally.

The areas located are: Porto de Riba, Figueiras, Area of the estuary and Lieiro quarry; we can not affirm the existence of other areas under new constructions, especially in the Porto de Riba and Figueiras.
\end{abstract}

Key words: Historic quarries, Galicia, Architectural Heritage, natural stone.

\section{ABRIDGED ENGLISH VERSION}

\section{Introduction}

Over the centuries the rocks have been used as natural materials of construction, thus defining a harmonious landscape, as the use of natural stone in small villages reflected the use of resources close to the places of 
Fernández Suarez, J., et al., 2017. Identificación, estudio preliminar y puesta en valor... Boletín Geológico y Minero, 128 (2): $485-498$

human settlement. Unlike current times, deposits exploited in small habited settlements, as the case of San Ciprián, were near of the buildings, because we have to consider that exploited rock volumes were not large, in most cases, as well as the absence of good roads of communication and means of transport for the distribution. After the location of the historical quarries of San Ciprián (Fernández Suárez et al., 2016), we have studied the characteristics and integration in the tourist catalogue of the village, taking advantage of the existing routes associated with the architectural heritage.

\section{Location, geographic features and geology}

San Ciprián is located on the coastal strip of the province of Lugo, in Galicia (43 ${ }^{\circ} 42^{\prime} 01,88^{\prime \prime} \mathrm{N} / 7^{\circ} 26^{\prime} 11,26^{\prime \prime}$ $W$, coordinates of the lighthouse). On the historical evolution of the village has much that see their geographic characteristics. On the one hand is the Atalaia, island where is located the settlement of Porto de Riba, which, at this moment, is linked to the mainland by a sandy isthmus. On the other hand in the continental part was installed, centuries later, a settlement known as Figueiras, placing Lieiro on the other side of the river Cobo.

With respect to geology the village is set on a granite massif, whose rocks present some variability (Tomás Fernández et al, 1977), in textural and mineralogical features, but in the area of study, this diversification is minimal.

\section{Areas of exploitation and historical quarries of San Ciprián and its association with the Architectural Heritage}

Some quarries and exploitation areas related to the local Architectural Heritage have been located in San Ciprián-Lieiro. These mining areas have the common characteristic of exploiting granite stone (rock used exclusively in the constructions) and being of small size (used for concrete and specific constructions), although one of them (Atalaia E).

\section{Area of Porto de Riba}

In this area four areas of stone extraction, Frentes $N$, the extraction area $E$, the quarry $S$ and the area of Cubelas, which comprise several temporary periods of operation, were located and investigated, and which served for the construction of Castro de La Atalaia, the village of Porto de Riba, the Chapel of San Ciprián and the salting factory.

\section{Area of Figueiras}

Although the urbanization of this area has been constant and important in the last five centuries, and there are probably hidden fronts under the buildings, it has been possible to verify the presence of two areas of exploitation of materials both to the $E$ and the $O$ of the settlement Figueiras, from which material was extracted for the construction of the town and the Chapel of San Andrés.

\section{Area of the estuary}

There are a number of zones of exploitation related to specific elements of heritage. It would therefore be open quarries in different periods aimed at covering the needs of a specific work, such as the Cantera de As Laxas, to build Os Pontons, the medieval bridge areas, for this infrastructure and the OTorno quarry for the Don Julián s factory.

\section{Zone of Lieiro}

The zone of extraction of the rocks for the construction of the Iglesia de Santa Maria de Lieiro is located very close to the temple. Part of the extraction area is missing due to the surrounding urbanization, but there are still signs of exploitation of stone material.

\section{Conservation proposal and use as a tourist resource.}

The extraction zones are located in the vicinity of the most notable constructions, so it would be interesting 
to put them in value, taking advantage of the accesses to them. In most cases the risk of disappearing of the localized marks is low, although in recent years we have witnessed the disappearance of certain examples by the urbanization of some areas (Frentes $N$ of Porto de Riba and As Laxas and Lieiro quarries), so its protection would be advisable.

The value proposition aims to take advantage of the synergy of the tourist and cultural offer of the village of San Ciprián, providing a new element very new with which it is possible to follow the temporal evolution in the extraction of natural stone, as well as the different techniques used throughout the centuries.

\section{Conclusions}

This study reveals the existence and characteristics of the signs of operation located in the historical quarries associated with the architectural heritage of San Ciprián. Taking advantage of the tourist attractions of the area, its monumental heritage and the proximity of the areas of extraction at these, intends to value through the creation of a didactic route in which to integrate the built heritage, geology and extraction activities, in order to make the relationships between built heritage and their environment to citizens and tourists.

\section{Introducción}

A lo largo de los siglos las rocas han sido utilizadas como materiales naturales de construcción, definiendo así un paisaje armónico, pues la utilización de piedra natural en pequeñas poblaciones reflejaba como poco la utilización de recursos cercanos a los lugares de asentamiento humano. A diferencia de épocas actuales, los yacimientos explotados en pequeños núcleos habitados, como era el caso de San Ciprián, estaban en las cercanías de las edificaciones, pues hay que considerar que los volúmenes de roca explotados no eran grandes, en la mayoría de los casos, así como la inexistencia de buenas vías de comunicación y de medios de transporte para la distribución de los mismos.

La localización de las canteras originales es importante, ya que permite obtener muestras de roca de características análogas a las del monumento (Fort, 2006), y con ello, evaluar su estado de deterioro y estimar la evolución en la vida útil del material, sobre la base de los resultados obtenidos en los ensayos de envejecimiento acelerado. También las muestras de rocas alteradas de forma natural en las canteras históricas pueden ser de utilidad para realizar con ellas los ensayos necesarios para seleccionar los tratamientos de consolidación e hidrofugación más adecuados. Esto únicamente puede ser útil si los procesos de deterioro y los daños causados son similares ya que, si no es así, irremediablemente tendremos que utilizar piedra procedente del mismo edificio.

Tras la localización de las canteras históricas de San Ciprián (Fernández Suárez et al., 2016), se estudian las características de las mismas y se propone su protección e integración en el catálogo turístico de la población, aprovechando las rutas existentes asociadas al Patrimonio Arquitectónico.

\section{Situación y características geográficas}

Localizada en la franja costera del $\mathrm{N}$ de la provincia de Lugo (Galicia), la población de San Ciprián $\left(43^{\circ} 42^{\prime} 01,88^{\prime \prime} \mathrm{N} / 7^{\circ} 26^{\prime} 11,26^{\prime \prime} \mathrm{O}\right.$, coordenadas del faro de San Ciprián), pertenece administrativamente al término municipal de Cervo, dentro de la comarca de La Mariña Occidental (Fig. 1). Desde el punto de vista eclesiástico se vincula a la parroquia de Santa María de Lieiro, diócesis de Mondoñedo, nota importante, pues gran parte del Patrimonio Monumental pertenece a edificaciones religiosas.

En la evolución histórica de San Ciprián tiene mucho que ver sus características geográficas. Por un lado se encuentra la Atalaia, isla donde se sitúa el asentamiento de Porto de Riba, que, en este momento, se encuentra unida a la parte continental por un tómbolo de arena. Actualmente, dicho tómbolo se

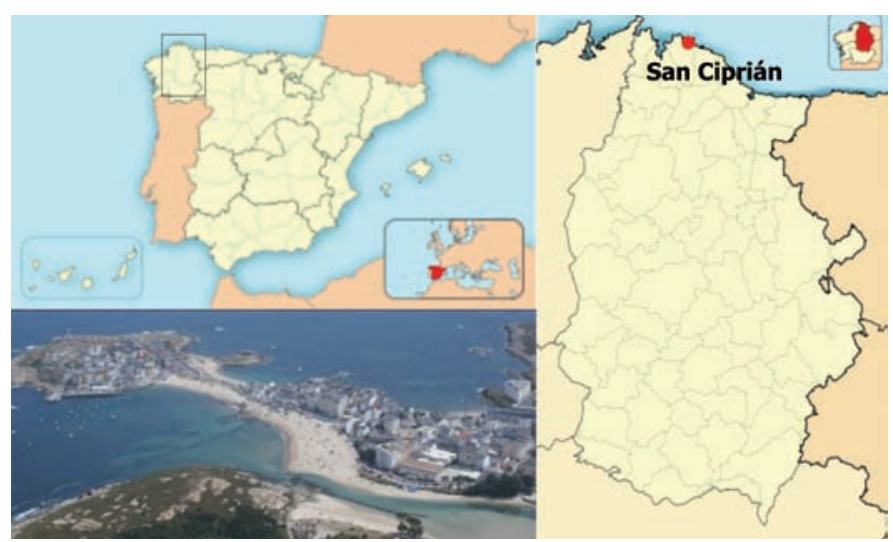

Figura 1. Situación de la localidad de San Ciprián, en la provincia de Lugo (Galicia, España).

Figure 1. Location of San Ciprián, in the province of Lugo (Galicia, Spain). 
encuentra fijado y con una carretera que conecta ambas partes, pero que en otros momentos antiguos el agua llegaba a cubrirlo, quedando aislado, ventaja aprovechada para la defensa por sus habitantes.

En la parte continental se instaló, siglos después, un asentamiento conocido como Figueiras, situándose Lieiro en la otra margen del río Cobo, como sede parroquial. Con el paso de los siglos estos asentamientos fueron creciendo, hasta la segunda mitad del siglo $X X$ cuando, tras la construcción de la fábrica de Alúmina-Aluminio, S.A., se crearon o ampliaron los asentamientos de La Veiga, Galiñeira, Galdín, etc., conformando lo que actualmente se conoce como San Ciprián. La zona de Lieiro, si bien no estuvo exenta de este desarrollo, experimentó un crecimiento moderado, incluso en épocas más reciente.

\section{Geología}

La población de San Ciprián se enclava sobre un macizo granítico, cuyas rocas presentan cierta variabilidad (Fernández Tomás et al, 1977), tanto a nivel textural como mineralógico, si bien en el ámbito de la zona de estudio esta diversificación es mínima.

Las rocas estudiadas, tanto en las áreas de extracción como en los monumentos asociados (Fig. 2), corresponden a leucogranitos de dos micas de tamaño de grano fino $y$, en ocasiones, muy fino. El color es gris, variando a tonalidades marrones por efecto de la alteración, en especial en las zonas cercanas a la costa, como ocurre en la zona de Figueiras.

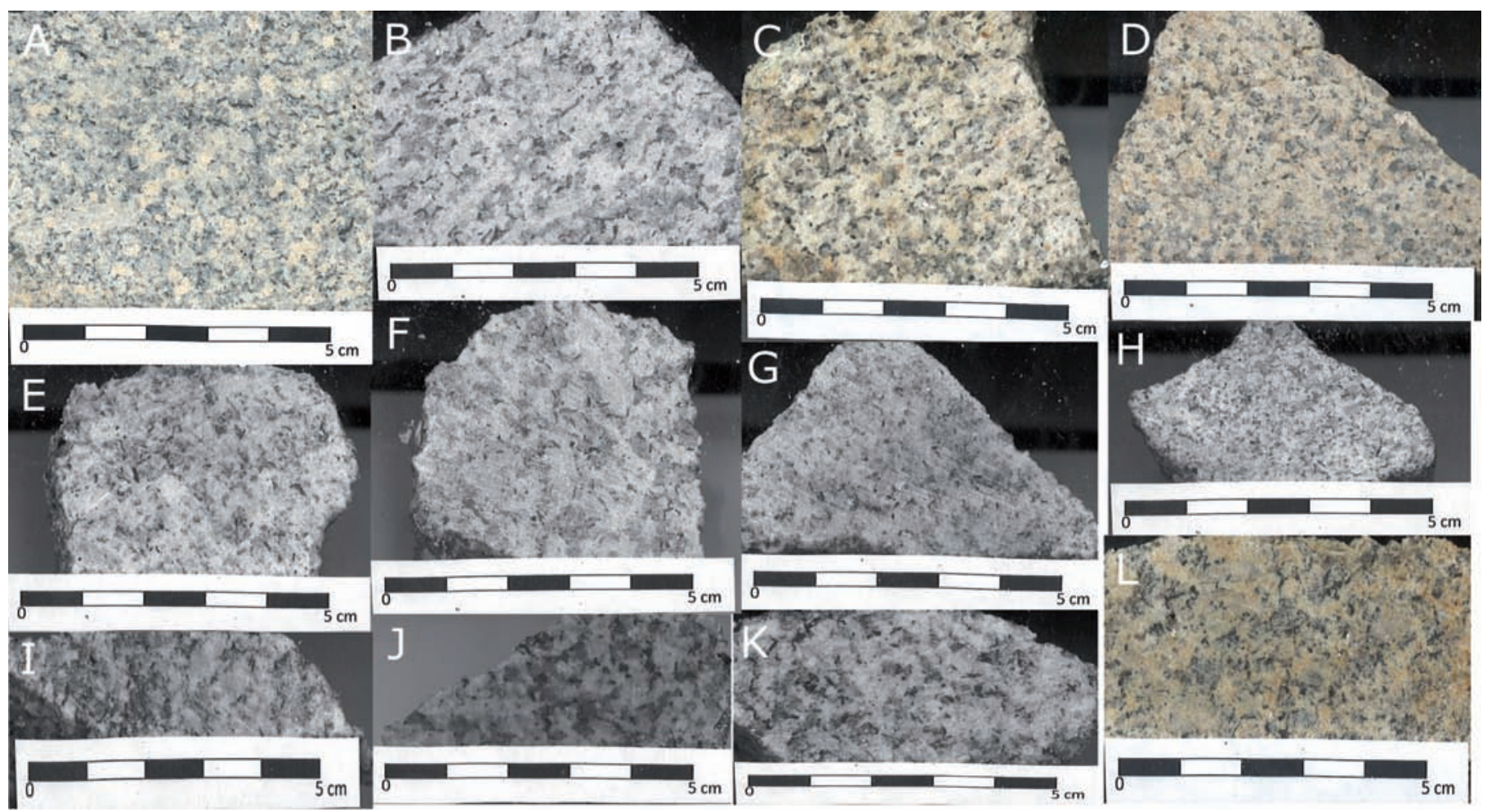

Figura 2. Muestras de granito recogidas en las áreas de extracción y en diferentes monumentos de San Ciprián. A- Muestra procedente del derribo parcial de una edificación del poblado de Figueiras. B- Muestra procedente del Área C del Área de extracción del puente medieval. C- Muestra procedente del Área de extracción Figueiras E. D- Muestra procedente de la Cantera de Lieiro. E- Muestra procedente del Área de extracción E de Porto de Arriba. F- Muestra procedente de la Cantera de Galdín. G- Muestra procedente del Castro de la Atalaia. H- Muestra procedente del Área B del Área de extracción del puente medieval. I- Muestra procedente de los Frentes N de Porto de Arriba. J- Muestra procedente de la Cantera de OTorno. K- Muestra procedente del aserradero y fábrica de salazón de Don Julián. L- Muestra procedente del Área de extracción de Cubelas.

Figure 2. Samples of granite collected in different extraction areas as well as in several monuments of San Ciprián. A- Sample from the partial demolition of a building in Figueiras. B-Sample from Area C, corresponding to one of the extraction area associated to the medieval bridge. C-Sample from the Extraction Area Figueiras E. D-Sample from the quarry of Lieiro. E-Sample from the Extraction Area E of Porto de Arriba. F- Sample from Galdín quarry. G-Sample from the Castro de la Atalaia. H-Sample from Area B corresponding to one of the extraction area associated to the medieval bridge. I- Sample from the Frentes $N$ of Porto de Arriba. J-Sample from the quarry of O Torno. K-Sample from the sawmill and Don Julián saltfish plant. L- Sample from the Cubelas extraction area. 
Apuntes históricos de la evolución del asentamiento de San Ciprián y elementos de su Patrimonio

\section{Arquitectónico}

La historia de este núcleo poblacional abarca varios siglos, desde los primeros pobladores conocidos, tribus celtas de los Egi-varri en la zona de la Atalaia, hasta la actualidad, pasando por los fenicios (siglo $\mathrm{V}$ a.C.), griegos (siglos II a.C.), romanos, suevos, árabes, normando, franceses e ingleses (Barro Quelle, 1989), si bien solo algunos de ellos han dejado su impronta en el Patrimonio Arquitectónico de San Ciprián.

Con la reciente aparición de un depósito de conchas y otros materiales arqueológicos durante la ejecución de unos movimientos de tierras para la construcción de edificaciones, se ha conseguido documentar la existencia de un poblado marítimo de tipología "castreña" (Fernández Rodríguez et al., 2008). Este asentamiento fue utilizado por tribus celtas $y$, posteriormente, asimilado por el imperio romano tras la conquista de Hispania, conocido en la actualidad como Castro de la Atalaia.

Tras una etapa de abandono (Xosé Ínsua, 2011), aunque si bien no se sabe si de una manera total, la zona fue repoblada con el nombre de San Ciprián en torno a los siglos VIII-IX, tras las reorganizaciones sufridas posteriores a las invasiones árabes.

La primera referencia documental de la que se dispone sobre San Ciprián (Sanctus Cyprianus, en latín) hace reseña a la localización de la ermita del mismo nombre, en Porto de Riba, citada en un documento en latín durante el reinado de Alfonso VII, el año 1128. Sin embrago la existencia de la misma podría ser anterior, ya que dentro de la capilla se encuentra una pila bautismal datada en un periodo que va del siglo VIII al X, dependiendo de los autores (Barro Quelle, 1989), realizada con material granítico de la zona.

$A$ partir de la segunda mitad del siglo $X V$, con un asentamiento de Porto de Riba sin espacio para ampliar, se comienza la edificación de la zona de Figueiras, en tierras de la Iglesia sin apenas producción agrícola. Este hecho, importante desde el punto de la extracción de piedra en esta zona, queda reflejado en los Archivos de la Catedral de Mondoñedo, donde las 29 casas construidas hasta 1629 fueron reclamadas por el Cabildo. Es en esta zona donde, en el año 1641, se funda el Hospital y Capilla de San Andes (Barro Quelle, 1989).

En este periodo es cuando se construye el puente medieval para unir la sede parroquial de Santa María de Lieiro y el pueblo de San Ciprián. Salva el río Cobo mediante un gran arco ojival, con calzada empedrada, y petriles de cantería unidos, en algunos casos, con grapas de hierro. Anteriormente a esta infraes- tructura, se utilizaba un efectivo y arcaico método conocido como "Os pontóns de As Laxas", que son unos grandes bloques de granito de superficie plana que quedan al descubierto durante la bajamar.

Desde la edad media fue un importante puerto ballenero hasta finales de la década de los años 60 del siglo XX, compartiendo esta notoriedad, sobre todo desde el siglo XIV, con los astilleros destinados a la construcción de carabelas y otras naves. De estas actividades se conservan dos conjuntos industriales, uno en la playa de Cubelas y otro en la margen izquierda de la desembocadura de la ría, donde se sitúa el aserradero y fábrica de salazón de Don Julián. La fábrica de salazón de pescado de Cubelas se sitúa en un saliente rocoso en las cercanías de Porto de Riba desde su emplazamiento a finales del siglo XIX, hasta su abandono en el año 1921, tras un incendio.

El crecimiento experimentado por la población fue lento y concentrado en dos zonas principales, como se desprende de la Carta particular de la ensenada y puerto de San Ciprián en la costa de la provincia de Lugo, levantada en 1859 (Riudavets, 1878). A lo largo de la primera mitad del siglo XX, San Ciprián fue creciendo de forma moderada, extendiendo su superficie por las zonas que bordean la ría, ganando terrenos a la rivera, con casa aisladas y pequeñas huertas anexas.

La explosión demográfica y constructiva en San Ciprián se produjo durante la segunda mitad del siglo XX. Si bien en un principio estuvo favorecida por la industria turística, fue con la instalación de la empresa Alúmina-Aluminio, S.A., actual factoría de Alcoa, a partir de la década de 1970 cuando la ampliación del pueblo produjo un gran cambio, aumentando en la primera década del siglo XXI.

\section{Áreas de explotación y canteras históricas de San Ciprián y su asociación con el Patrimonio Arquitectónico}

Se han localizado en la población de San CipriánLieiro una serie de canteras y zonas de explotación relacionadas con el Patrimonio Arquitectónico local. Estas explotaciones tienen la característica común de explotar piedra granítica (roca utilizada en exclusiva en las construcciones) y ser de pequeño tamaño (utilizadas para unas construcciones concretas y puntuales), si bien una de ellas (Atalaia E) se llegó a explotar de manera semi-industrial, suministrando losas, para el empedrado de calles, a ciudades tan distantes como Oviedo, en Asturias (Gutiérrez Claverol et al., 2012). 


\section{Zona de Porto de Riba}

En esta zona se localizaron e investigaron cuatro áreas de extracción de piedra, Los Frentes $\mathrm{N}$, el área de extracción $\mathrm{E}$, la cantera $\mathrm{S}$ y la zona de Cubelas, que comprenden varios periodos temporales de funcionamiento (Fig. 3).

\section{Frentes $N$}

Los denominados Frentes $\mathrm{N}$, situados en el extremo septentrional de Punta Atalaia, se encuentran actualmente cubiertos por arenas y vegetación, habiendo perdido la antigua morfología de los frentes de cantera que existían hasta la remodelación de la zona y la construcción del mirador. No obstante se han reconocido varias señales de explotación en pequeños afloramientos graníticos, consistentes en escalonamientos, marcas de incisiones de cuñas y un bloque parcialmente trabajado y prácticamente cubierto (Fig. 4).

\section{Área de extracción $E$}

En el extremo este aparecen numerosas señales de utilización de piedra y algún bloque suelto, sin embargo las marcas localizadas tienen características modernas (siglos XIX y XX), constando de grandes cuñas y barrenos (Fig. 5), así como una zona de acopio y/o trabajo a lo largo del límite de la costa este de Punta Atalaia, que prácticamente llega hasta la fábrica de salazón de Cubelas. La distribución de las mar-

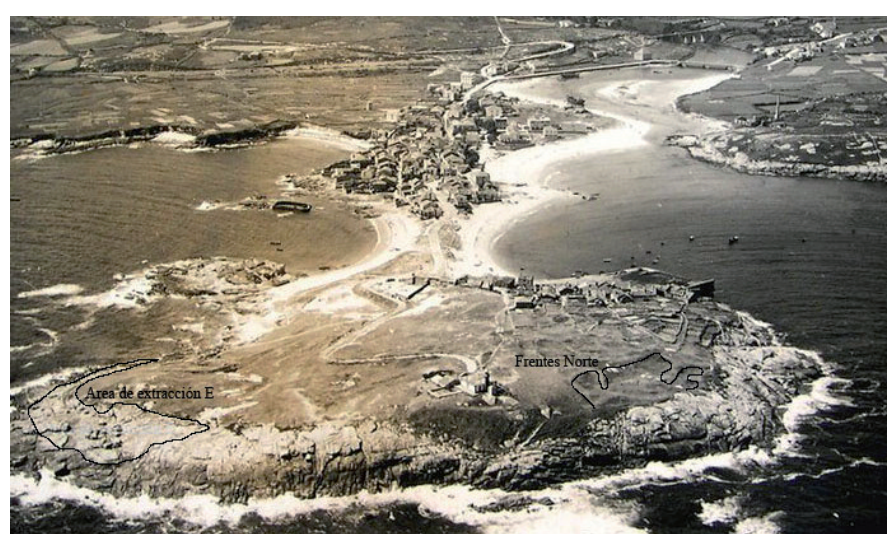

Figura 3. Fotografía aérea de las zonas de extracción de piedra de construcción en el Área de Porto de Arriba. A la derecha los Frentes $\mathrm{N}$ y a la izquierda el Área de extracción E.

Figure 3. Aerial photography of the extraction areas of building stones in the Area of Porto de Arriba. On the right: Frentes N; On the left: Extraction Area $E$.

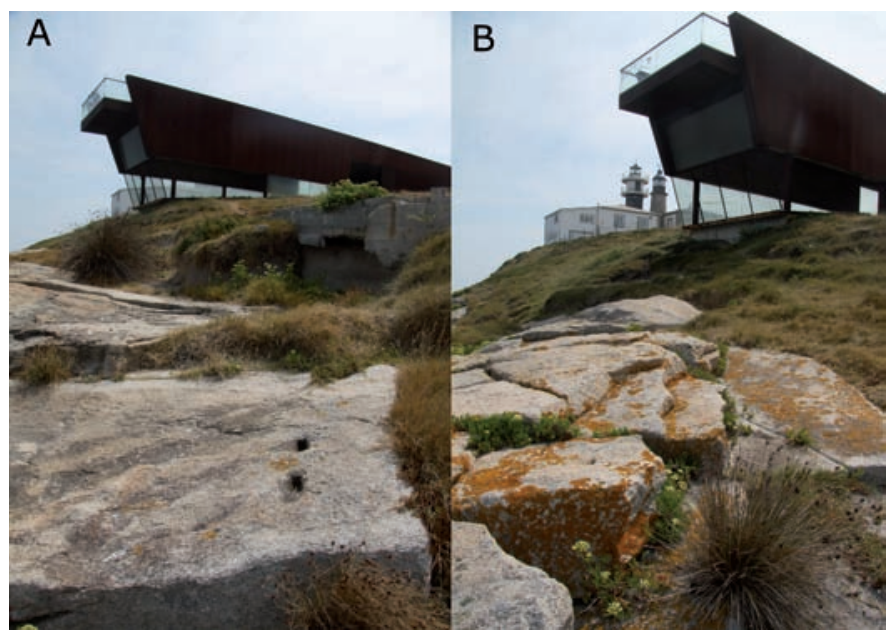

Figura 4. A- Marcas de cuñas. B- Frentes escalonados para la extracción de piedra y marcas de utilización de cuñas.

Figure 4. A-Wedge marks. B- Quarry fronts and wedge marks.

cas localizadas es bastante irregular, pues en la parte norte de la zona se localizan cuñas pero no barrenos, siendo a la inversa en la parte cercana a la fábrica de salazón.

Estas zonas de aprovechamiento (Frentes N y Área

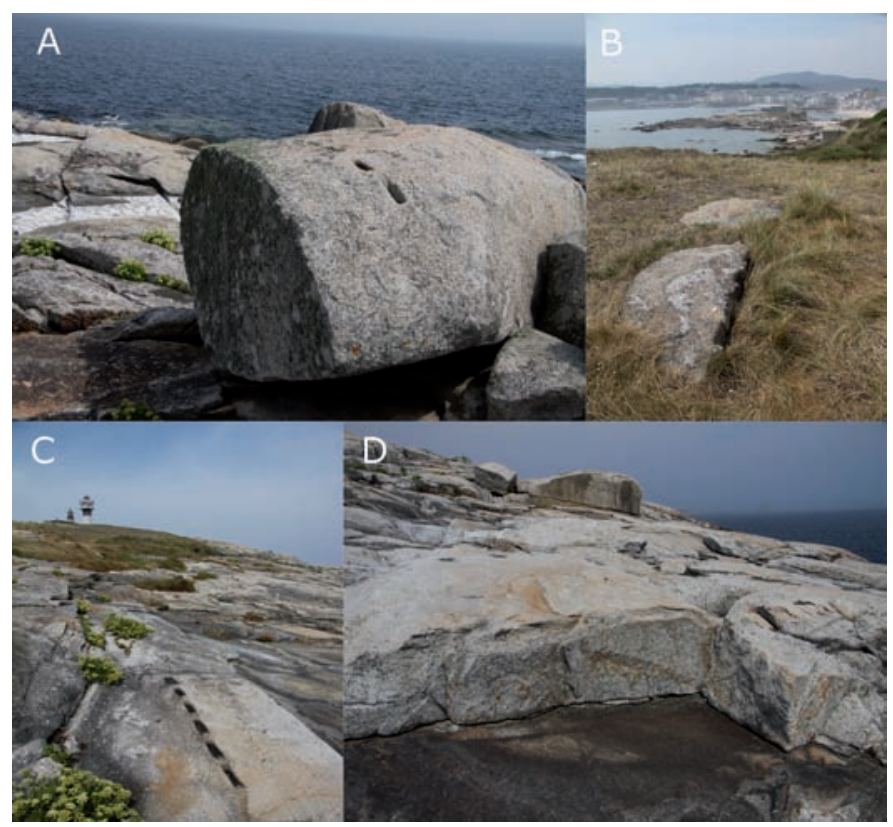

Figura 5. A- Marcas de cuñas en un bloque no extraído. B- Marcas de cuñas en una zona de extracción de bloque. C- Marcas alineadas de cuñas industriales. D- Frente de extracción de bloques.

Figure 5. A-Wedge marks in a non-extracted block. B-Wedge marks in a extraction zone. $C$ - Aligned marks of industrial wedges. $D$ Block extraction front. 
de extracción E) corresponderían, principalmente, a las primeras fases de construcción del castro, en época celta, y su ampliación en época romana. Complementaría la utilización de bloques naturales recogidos de las zonas cercanas a la costa, que son observables actualmente en los muros de las edificaciones, donde aparecen redondeados por la acción del aire y agua.

También sería fuente de extracción de materiales para la construcción de la Capilla de San Ciprián, por no estar fijado el tómbolo de arena en su época de construcción y las dificultades que el traslado de sillares por la arena podía suponer. Sin embargo, para esta construcción no hay que obviar el fuerte contenido de reutilización de materiales del castro, que surtiría de materia prima para nuevas construcciones, lo que refleja la ausencia de sillares y rocas labradas en la construcción, especialmente en época romana.

La utilización del Área de extracción E en siglos recientes está relacionada con una explotación y comercio de materiales de manera semi-industrial, siendo de esta zona de donde se extraían las placas que fueron exportadas a la ciudad de Oviedo para su empedrado, aprovechando la cercanía del puerto para su carga y transporte.

\section{Área de extracción de Cubelas}

Como continuación natural de los afloramientos del Área de extracción $\mathrm{E}$, en los alrededores de la fábrica de salazón aparecen un gran número de vestigios de extracción de rocas, relacionadas con la construcción de este complejo. Son marcas muy variadas, apareciendo en un espacio físico muy reducido sillares adosados al macizo, marcas de cuñas, rozas y bloques sueltos parcialmente labrados, aunque estos últimos pueden ser producto del desmantelamiento de la edificación.

\section{Cantera S}

La zona que plantea más problemas es la situada en la parte S de Punta Atalaia, actualmente tapada con materiales de excavación de construcciones de la segunda mitad del siglo XX, siendo imposible identificar afloramientos naturales. Según la documentación consultada (Barro Quelle, 1989), en esta zona se encontraba una plaza donde, desde la Edad Media, eran descuartizadas las ballenas. Esta plaza de cantera, y el talud por el que discurre la carretera que va al faro, constituirían la zona de explotación de materiales utilizados para la posible construcción de parte de la capilla de Porto de Riba y las construcciones de casas particulares durante esta época. Sin embargo la fuerte removilización de materiales, durante la construcción reciente de las casas, impide asegurar totalmente el origen de las rocas.

\section{Zona de Figueiras}

A pesar de que la urbanización de esta área ha sido constante e importante en los últimos cinco siglos, y probablemente haya frentes ocultos bajo las construcciones, se ha podido constatar la presencia de dos zonas explotación de materiales tanto al E como al $\mathrm{O}$ del asentamiento de Figueiras, de donde se extrajo material para la construcción del poblado y la Capilla de San Andrés.

El Área de extracción E (Figueiras E) es donde mejor se puede apreciar la explotación de rocas para la construcción (Fig. 6A y $6 \mathrm{C}$ ), pudiendo observarse una evolución temporal de la misma desde métodos artesanales (cuñas) hasta más modernos (mediante la presencia de barrenos para precortar los bloques) (Fig. 6B). Estos métodos de extracción indican que esta zona estuvo en funcionamiento desde la época medieval hasta bien entrado el siglo XX.

El método más tradicional es la utilización de cuñas para el precorte de los bloques, aunque no hay referencias a si el material utilizado fue la madera o el metal. Lo que sí se puede observar es la oxidación de las oquedades de las cuñas, lo que nos lleva a pensar en la utilización de este segundo material. En la actualidad se puede observar un bloque $(2 \times 1 \times 1 \mathrm{~m})$ con signos claros de extracción mediante este método, que no llegó a completarse (Fig. 6E). Hay otros bloques, de más pequeño tamaño $(1 \times 0,5 \times 0,5 \mathrm{~m})$, con marcas de extracción de corte mediante cuñas (Fig. $6 D)$, que fueron abandonados tras su separación del macizo. En épocas más recientes, la utilización de barrenos para la explotación de esta zona ha estado más extendida. Son numerosas las marcas de perforación presentes en el área, tanto en bloques aislados como en el mismo terreno.

Se ha constatado, además, la presencia de una "lajera", zona para la extracción de lajas de granito que, antes de la llegada de la pizarra de techar, servían para el recubrimiento de las viviendas de la zona (Fig. 6F).

Aprovechando la característica natural de alteración de los granitos (cortezas de meteorización), la extracción de este material se realizaba manualmente, obteniéndose material de formas irregulares y tamaños variables. Se han observados dimensiones importantes de unos $40 \times 30 \mathrm{~cm}$ y grosores de entre 3 


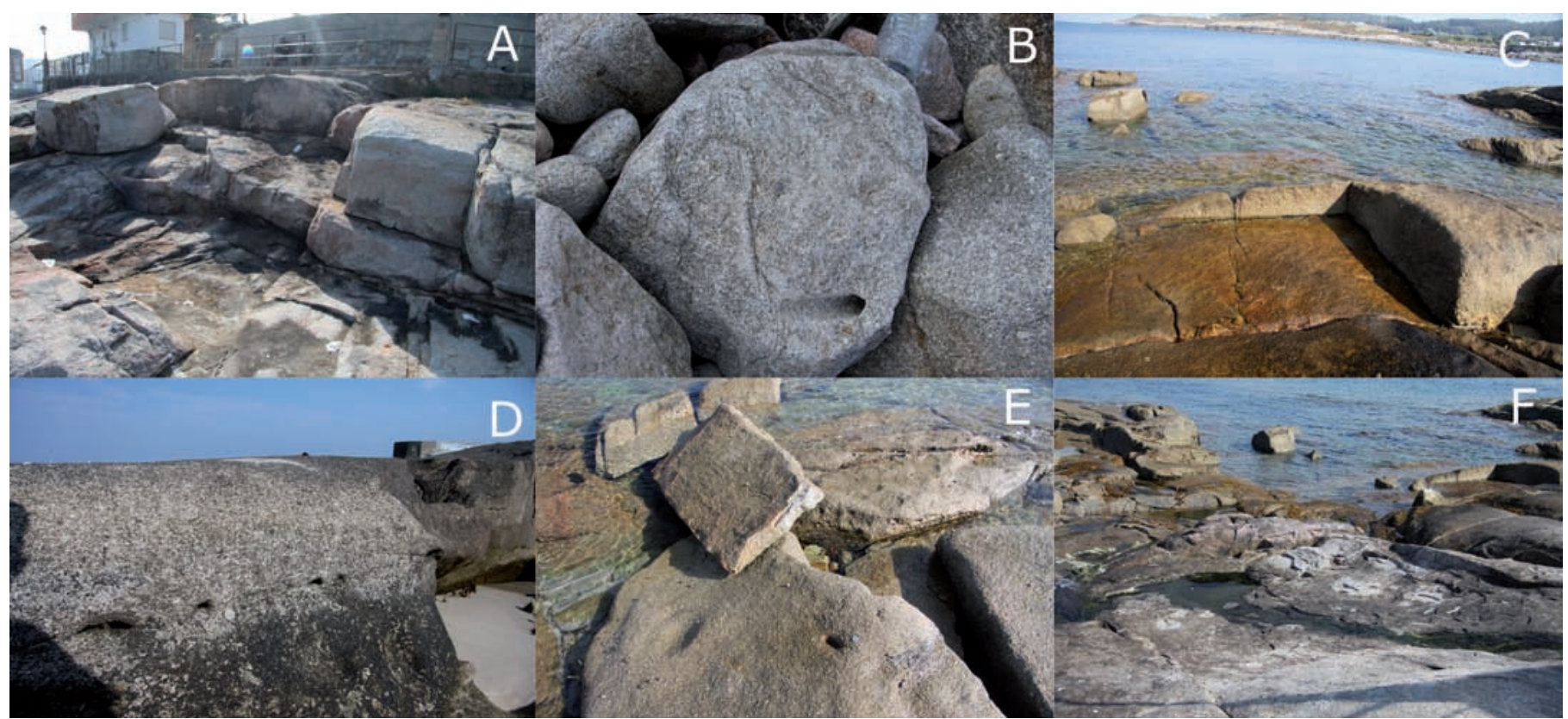

Figura 6. A- Frente de extracción de bloques. B- Marcas de barrenos. C- Frente de extracción de bloques. D- Marcas alineadas de cuñas. E- Bloque extraído en una zona de explotación. F- Aspecto que presenta la lajera en la actualidad.

Figure 6. A-Block extraction front.B-Borehole. C-Block extraction front. D-Aligned wedge marks. E- Extracted block in a extraction area. F-Appearance of "La lajera" extraction area nowadays.

y $6 \mathrm{~cm}$, en los restos que actualmente son observables.

En el Área de extracción O (Figueiras O) aflora un plutón granítico, donde se sitúa el monumento a la Marina de Guerra, donde aparecen signos de explotación, a modo de barrenos, sin que se haya podido constatar la presencia de marcas de extracción artesanal. Hay acumulados numerosos bloques de granito en la zona, sin embargo el derribo de unas casas situadas junto a esta zona en la década de 1990, nos impide asegurar que la totalidad de los mismo provenga de esta zona de explotación.

\section{Zona de la ría}

En esta zona se sitúan una serie de áreas de explotación relacionadas con elementos concretos del patrimonio. Se trataría por tanto de canteras abiertas en diferentes periodos encaminadas a cubrir las necesidades de una obra en concreto, siendo su reutilización muy limitada, salvo en el caso de la Cantera de As Laxas, pues las frecuentes reparaciones de estos elementos implicaría su uso continuado a lo largo de los siglos. Barro Quelle (1989) ya señala la obligación de los canteros de las poblaciones cercanas a acudir a este punto para la reparación de los pasos, por medio de bandos dictados por los alcaldes.

\section{Cantera de OTorno}

Frente a la playa de O Torno, al otro lado de la ría, existe una cantera de extracción de piedra para construcción, que estuvo en funcionamiento hasta mitad del siglo XX (Fig. 7A). En la actualidad son visibles claramente tanto el frente como la plaza de cantera, así como algunos bloques en la zona (Fig. 7B). La extracción, según los restos observables en la actualidad, era realizada de forma artesanal mediante cuñas, sin marcas de barrenos. La cercanía de la Fábrica de Salazón y Aserradero de la ría, que permitía el transporte de los sillares de granito, así como la similitud de las rocas del afloramiento y los muros indican la procedencia de las mismas.

\section{Cantera de As Laxas}

En la zona de comienzo de los "pasos" de la margen izquierda de la ría era observable, hasta no hace muchos años, un antiguo frente de extracción de unos 2 o $3 \mathrm{~m}$ de altura y hasta $20 \mathrm{~m}$ de anchura. De esta zona, debido a la proximidad, fueron extraídos los "pasos" a lo largo de los siglos, así como materiales para las construcciones de edificaciones de la zona. En la actualidad la zona ha desaparecido bajo nuevas edificaciones y remodelaciones del entorno 


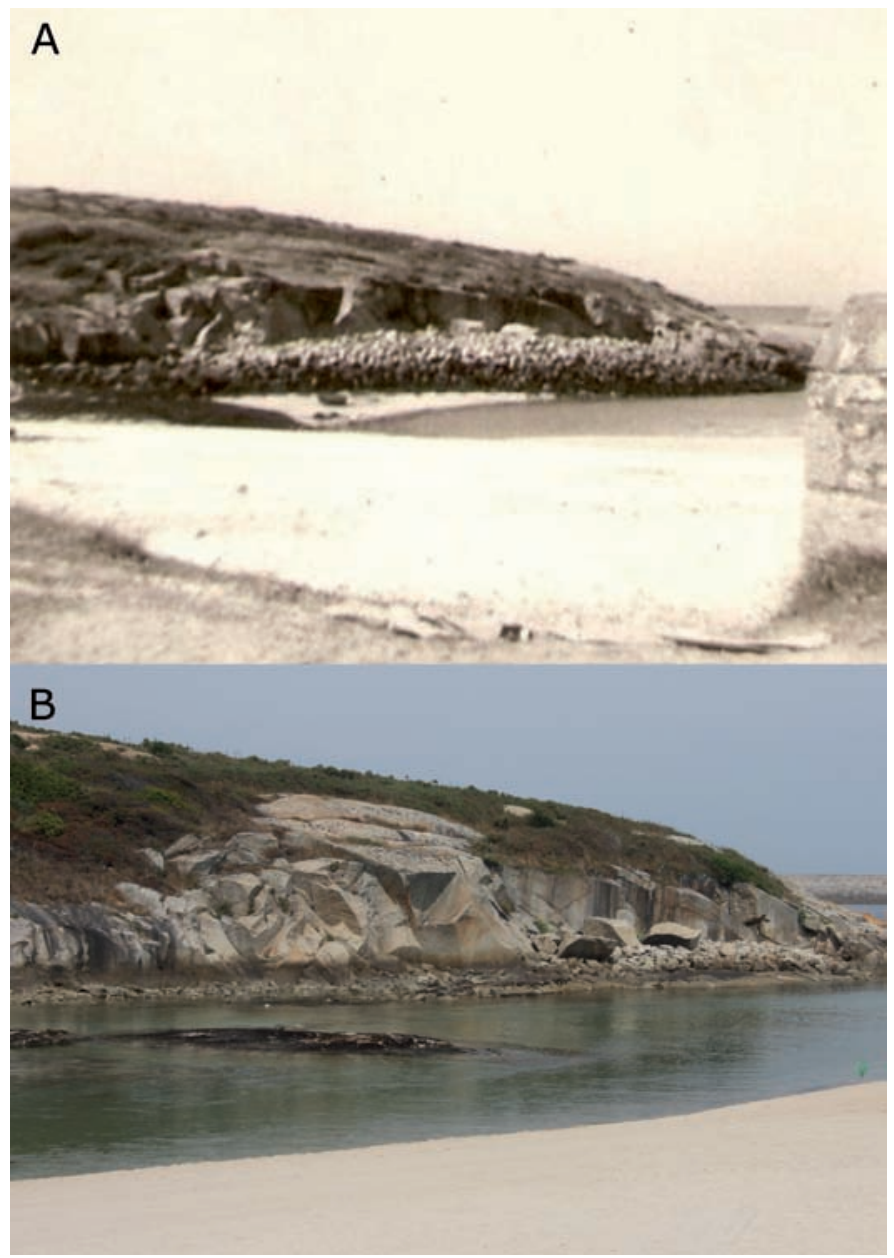

Figura 7. A- Cantera de OTorno en una fotografía tomada en tono a la década de 1940. B- Aspecto que presenta la cantera actualmente. Figure 7. A- O Torno quarry in a photograph taken in the 1940s. BCurrent appearance of the quarry.

(Fig. 8B). En la Fig. 8A, datada en la segunda mitad del siglo $X X$, aún es posible reconocer el frente de canteras antes de su desaparición.

\section{Área de extracción del puente medieval}

Se han localizado tres zonas de explotación de piedra de construcción distribuidas en ambos márgenes del río. Por proximidad al puente y volumen de roca explotada, así como la ausencia en la zona de otras construcciones, es más que probable su origen como fuente de abastecimiento de material para la construcción de la obra civil, permaneciendo las marcas localizadas intactas de la construcción de la misma.

El área $A$ se encuentra en la margen izquierda del río, donde aparece una serie de afloramientos roco- sos que fueron aprovechados para la extracción de sillares. En la actualidad es posible observar varias marcas de cuñas (Fig. 9A), perfectamente alineadas, como paso previo a la operación de corte, así como restos de extracción de bloques. Es interesante el tamaño de las cuñas, pues asumiendo que son originales de la Edad Media, nos permite conocer las dimensiones aproximadas de las cuñas empleadas en ese periodo.

Las Áreas B y C se sitúan en la margen derecha del río, separadas una de otra unos $50 \mathrm{~m}$, no habiéndose localizado ningún signo de explotación entre ellas. En el Área B son observables varios bloques sueltos, así como como bancos (Fig. 9B), marcas de cuñas y rozas y segmentaciones de granito en los frentes. En el Área $\mathrm{C}$ aparecen bancos de explotación escalonados (Fig. 9C), así como una trinchera (Fig. 9D) recubierta de escombro y vegetación de lo que pudo haber sido otro frente cantera.

\section{Zona de Lieiro}

La zona de extracción de las rocas para la construcción de la Iglesia de Santa María de Lieiro se encuentra situada muy cerca del templo. Parte de la zona de extracción, especialmente la gran plaza de cantera, se encuentra desaparecida por la urbanización del entorno, pero aún son observables signos de explotación de material pétreo.

Hasta hace relativamente poco tiempo, por lo menos hasta los años 20 del siglo XX, se podía ver la antigua cantera (Fig. 10), con varios frentes y bancos de extracción, que seguramente fueran activados en diferentes épocas para otras construcciones, como por ejemplo la casa sacerdotal, también en las cercanías.

Actualmente son visibles algunas zonas de afloramiento con marcas de extracción de piedra en una de las fincas no construidas, así como bancos escalonados junto a los muros de una de las nuevas edificaciones.

\section{Área de Galdín}

La antigua cantera de Galdín, hoy parcialmente desaparecida por las obras de desmonte de nuevas edificaciones aunque visitable, estuvo en funcionamiento a partir de la década de 1970; aunque, en cualquier caso, no antes de 1956 como refleja la fotografía aérea del vuelo americano de este año. La utilización de las rocas de esta cantera está determinada por la expansión de la población de San Ciprián a partir de 


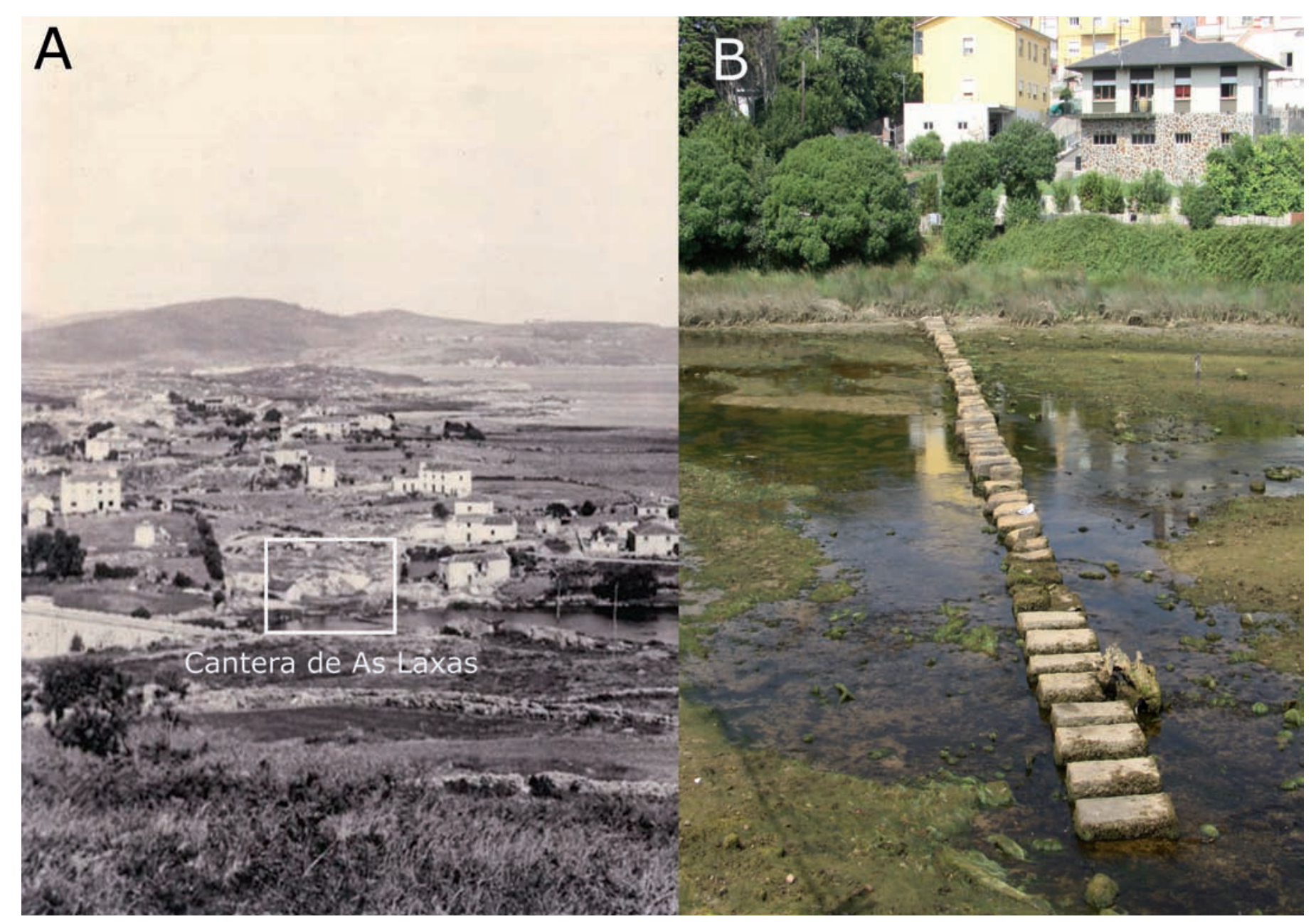

Figura 8. A- Frente de explotación de la Cantera de As Laxas antes de su desaparición. B-Aspecto que presentan en la actualidad Os Pasos, en cuyo final se situaba la cantera hoy desaparecida.

Figure 8. A- Extraction front of the As Laxas quarry before its disappearance. B-Current appearance of Os Passos. The old quarry was located at the end of the river crossing.

esa década con la implantación del complejo fabril de Alúmina-Aluminio, S.A., y la necesidad de construcción de viviendas para los trabajadores.

No hay constancia de la utilización de las rocas de esta cantera en ningún elemento patrimonial del pueblo, pero marca el fin de la explotación de piedra de construcción en la zona de San Ciprián, que se había extendido desde hacía más de dos milenios. Esta falta de relación directa con los elementos constituyentes del Patrimonio Arquitectónico y la similitud de la roca explotada con los otros tipos petrológicos empleados como material de construcción en la zona, la hace relevante en el sentido de poder ser fuente de material para la realización de análisis y ensayos de deterioro y como fuente de material para futuras restauraciones, por lo que su conservación parcial sería interesante.

\section{Propuesta de conservación y su utilización como recurso turístico}

Las zonas de extracción se encuentran en las inmediaciones de las construcciones más notables de la localidad, por lo que sería interesante su puesta en valor, aprovechando los accesos a las mismas. En la mayoría de los casos el riesgo de desaparición de las marcas localizadas es bajo, aunque en los últimos años hemos asistido a la desaparición prograsiva de algunos ejemplos por la urbanización de algunas zonas (Frentes $\mathrm{N}$ de Porto de Riba y Cantera de As Laxas y Lieiro), por lo que su protección sería recomendable.

La propuesta de puesta en valor va encaminada a aprovechar la sinergia de la oferta turística y cultural de la población de San Ciprián, proveyendo de un 


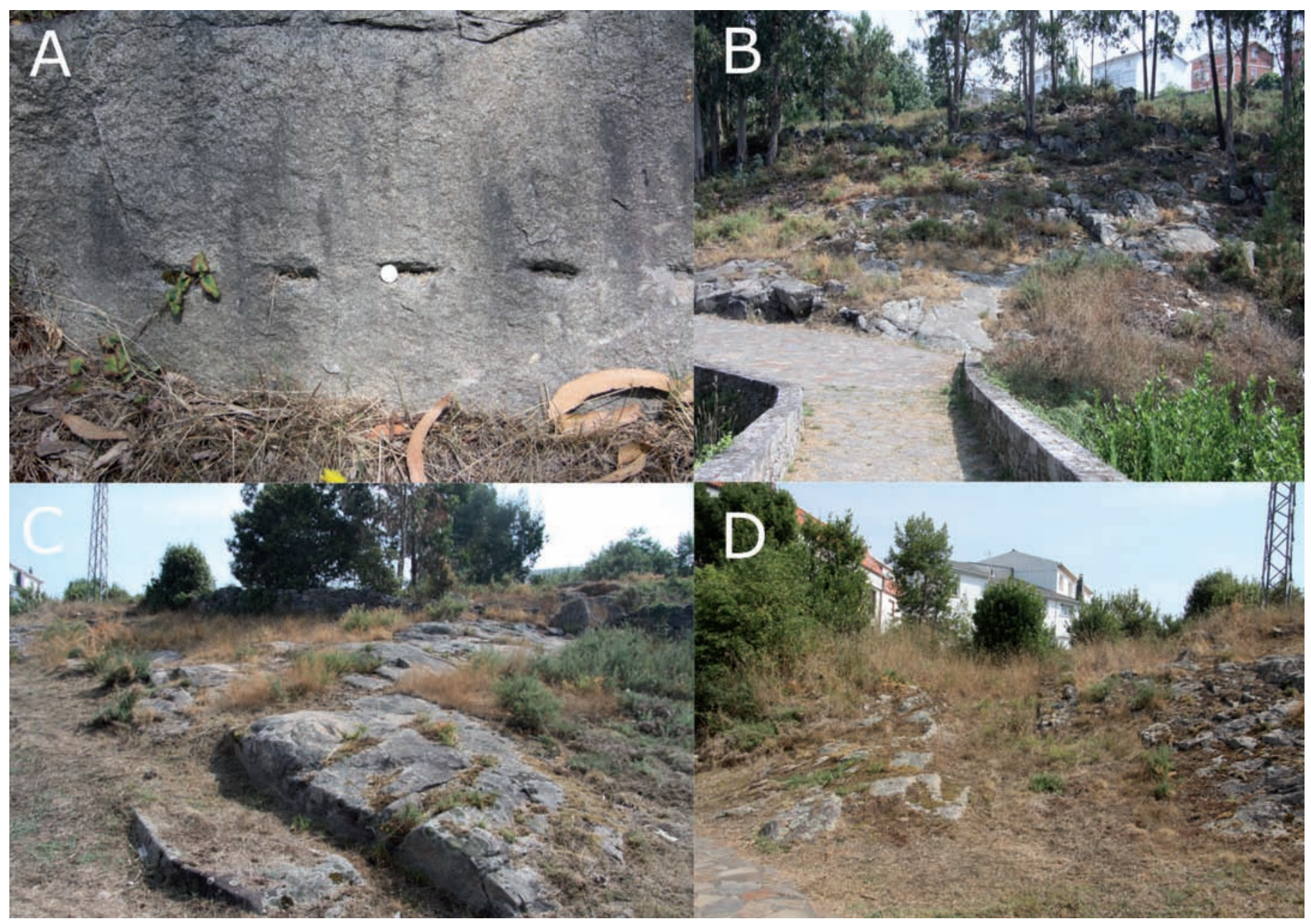

Figura 9. A- Marcas alineadas de cuñas en el Área A. B- Aspecto general de la zona de extracción del Área B. C- Bancos escalonados de extracción en el Área C. D- Antigua trinchera, parcialmente tapada en el Área C.

Figure 9. A-Aligned wedge marks in Area A. B-General appearance of the extraction area B. C-Quarry fronts in Area C. D-partially covered trenchin Area $C$.

nuevo elemento muy novedoso con el que se puede seguir la evolución temporal en la extracción de piedra natural, así como las diferentes técnicas utilizadas a lo largo de los siglos.

Toda esta actividad extractiva ha evolucionado con el crecimiento urbanístico de la localidad, y la necesidad de material para sus construcciones, siendo una actividad histórica que, de no ponerse en valor y acercarla a los ciudadanos y visitantes, puede caer en el olvido.

Además, la utilización de las canteras históricas como recurso didáctico original y poco convencional en la enseñanza de las ciencias geológicas puede incentivar la curiosidad de alumnos, aprendiendo a observar y a deducir aspectos interesantes sobre la geología local (Varas et al., 2010). Siendo la iniciativa que en este trabajo se propone una ocasión para edu- car y generar una sensibilidad hacia el patrimonio heredado y su entorno en las nuevas generaciones, a la vez de conocer los materiales geológicos empleados en la construcción de los edificios singulares de la localidad, que presentan un gran potencial didáctico, como así se señaló en el I Congreso Español de Geología celebrado en Segovia (García-Ruiz, 1986).

De este modo, mediante la creación de una ruta a pie se integraría el patrimonio tradicional adaptado al entorno más inmediato, con la geodiversidad y el patrimonio geológico que en la zona presenta una gran relevancia, y del que se ha obtenido el recurso esencial para la construcción de las edificaciones, todo ello con una fuerte connotación didáctica relacionado con el término de Geoturismo (Hose, 2000).

Esta ruta, adecuadamente señalizada y completada con la incorporación de paneles didácticos en las 


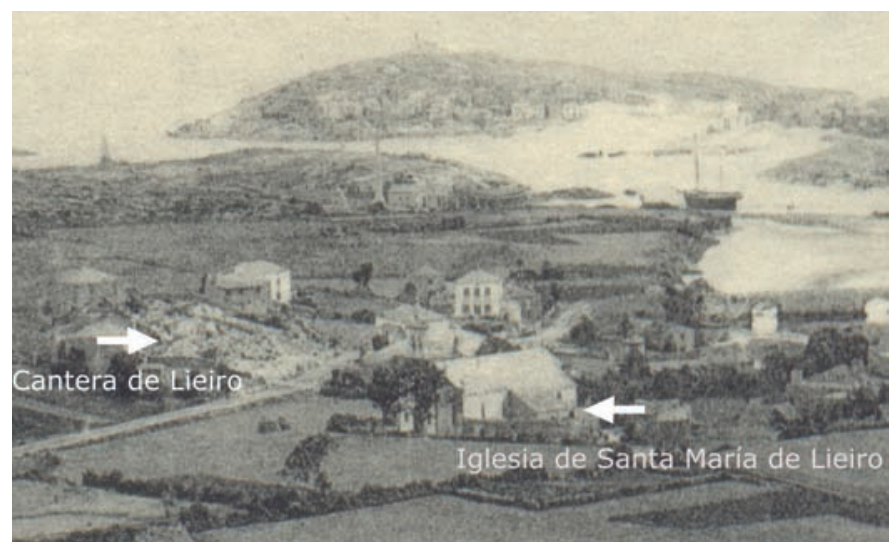

Figura 10. Fotografía de Primitivo Hernández Sanpelayo, de 1915, procedente la publicación Criaderos de Hierro de Galicia (Hernández Sampelayo, 1935), donde se observa la antigua cantera de Lieiro, junto a la Iglesia parroquial de Santa María.

Figure 10. Photography by Primitivo Hernández Sanpelayo, (1915) included in the publication of Criaderos de Hierro de Galicia (Hernández Sampelayo, 1935). The old quarry of Lieiro can be seen, next to the church of Santa María. áreas anteriormente expuestas, participaría de forma activa en la divulgación del entorno, de las ciencias geológicas y un mejor conocimiento del Patrimonio Arquitectónico local, y por tanto en su conservación (Fig. 11).

La tabla 1 señala los principales signos de explotación observables en las diferentes áreas, así como los elementos asociados a las mismas.

\section{Conclusiones}

A partir de este estudio se pone de manifiesto la existencia y las características de los signos de explotación localizados en las canteras históricas asociadas con el Patrimonio Arquitectónico de San Ciprián, si bien en algunos casos han desaparecido parcial o totalmente. No se puede afirmar la inexistencia de otras zonas de extracción actualmente desaparecidas, especialmente en la Zona de Porto de Riba y

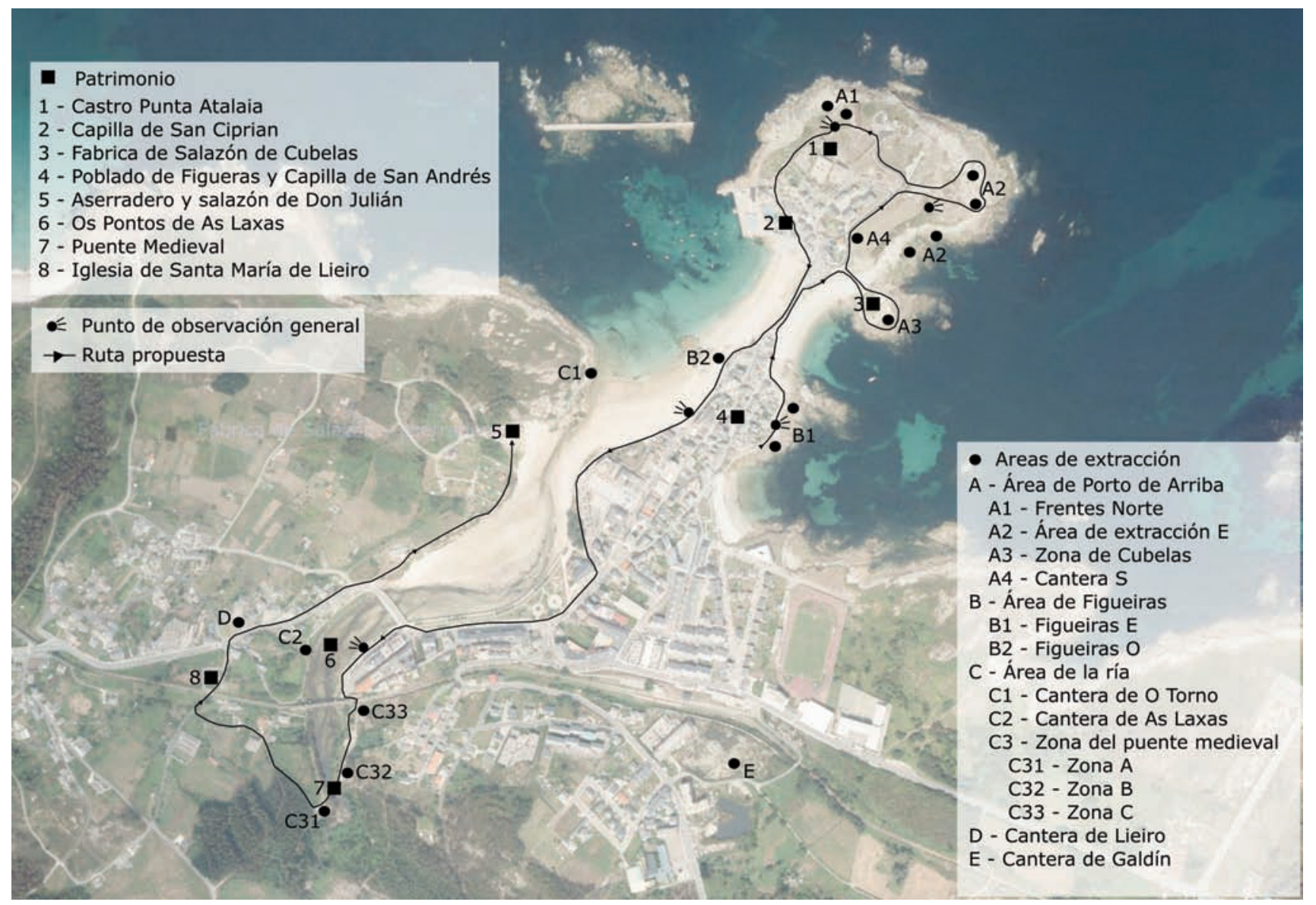

Figura 11. Situación del patrimonio monumental, zonas extracción de piedra de construcción identificadas y la posible ruta para la observación de ambas.

Figure 11. Location map of monumental heritage, areas of stone extraction and proposed visit route. 
Fernández Suarez, J., et al., 2017. Identificación, estudio preliminar y puesta en valor... Boletín Geológico y Minero, 128 (2): $485-498$

\begin{tabular}{|c|c|c|c|}
\hline Área / Area & Zona / Zone & Elemento asociado / Associated item & Marcas visibles / visible marks \\
\hline \multirow{4}{*}{$\begin{array}{l}\text { Zona de Porto de Riba } \\
\text { Zone of Porto de Riba }\end{array}$} & $\begin{array}{l}\text { Frentes } \mathrm{N} \\
\text { Fronts } \mathrm{N}\end{array}$ & $\begin{array}{l}\text { Castro de Punta Atalaia } \\
\text { Castro of Punta Atalaia } \\
\text { Capilla de San Ciprián } \\
\text { Chapel of San Ciprián }\end{array}$ & $\begin{array}{l}\text { Bancos escalonados, sillares abandonados y cuñas } \\
\text { staggered benches, abandoned ashlars and wedges marks }\end{array}$ \\
\hline & $\begin{array}{l}\text { Área de extracción } \mathrm{E} \\
\text { Extraction área } \mathrm{E}\end{array}$ & $\begin{array}{l}\text { Castro de Punta Atalaia } \\
\text { Castro of Punta Atalaia } \\
\text { Capilla de San Ciprián } \\
\text { Chapel of San Ciprián }\end{array}$ & $\begin{array}{l}\text { Bancos escalonados, extracción de bloques, sillares adosados al macizo, sillares } \\
\text { abandonados, rozas y cuñas } \\
\text { Staggered benches, extraction of blocks, ashlars attached to the massif, abandoned ashlars, chafe and } \\
\text { wedges marks }\end{array}$ \\
\hline & $\begin{array}{l}\text { Cantera S } \\
\text { Quarrys }\end{array}$ & $\begin{array}{l}\text { Castro de Punta Atalaia } \\
\text { Castro of Punta Atalaia } \\
\text { Capilla de San Ciprián } \\
\text { Chapel of San Ciprián }\end{array}$ & $\begin{array}{l}\text { Cantera desaparecida } \\
\text { Missing quarry }\end{array}$ \\
\hline & $\begin{array}{l}\text { Zona de Cubelas } \\
\text { Zone of Cubelas }\end{array}$ & $\begin{array}{l}\text { Fábrica de salazón } \\
\text { Salting factory }\end{array}$ & $\begin{array}{l}\text { Bancos escalonados, extracción de bloques, sillares adosados al macizo, sillares } \\
\text { abandonados, rozas y cuñas } \\
\text { Staggered benches, extraction of blocks, ashlars attached to the massif, abandoned ashlars, chafe and } \\
\text { wedges marks }\end{array}$ \\
\hline \multirow[t]{2}{*}{$\begin{array}{l}\text { Zona de Figueiras } \\
\text { Zone of Figueiras }\end{array}$} & $\begin{array}{l}\text { Figueiras } \mathrm{E} \\
\text { Figueiras E }\end{array}$ & $\begin{array}{l}\text { Capilla de San Andrés } \\
\text { Chapel of San Andrés } \\
\text { Poblado de Figueiras } \\
\text { Village of Figueiras }\end{array}$ & $\begin{array}{l}\text { Extracción de bloques, cuñas, barrenos, "Iajera" y bloques abandonados } \\
\text { Extraction of blocks, chafe and drill marks, exfoliation of granite area and abandoned bloks }\end{array}$ \\
\hline & $\begin{array}{l}\text { Figueiras O } \\
\text { Figueiras W }\end{array}$ & $\begin{array}{l}\text { Poblado de Figueiras } \\
\text { Village of Figueiras }\end{array}$ & $\begin{array}{l}\text { Extracción de bloques y bloques abandonados } \\
\text { Extraction of blocks and abandoned bloks }\end{array}$ \\
\hline \multirow{3}{*}{$\begin{array}{l}\text { Zona de la Ría } \\
\text { Zone of the estuary }\end{array}$} & $\begin{array}{l}\text { Cantera de O Torno } \\
\text { O Torno quarry }\end{array}$ & $\begin{array}{l}\text { Aserradero y fábrica de salazón de Don } \\
\text { Julián } \\
\text { Don Julián's sowmill and salting factory }\end{array}$ & $\begin{array}{l}\text { Frente de cantera y bloques abandonados } \\
\text { Quarry front and abandoned bloks }\end{array}$ \\
\hline & $\begin{array}{l}\text { Canteras de As Laxas } \\
\text { As Laxas quarry }\end{array}$ & $\begin{array}{l}\text { Os Pasos } \\
\text { Os Pasos }\end{array}$ & $\begin{array}{l}\text { Cantera desaparecida } \\
\text { Missing quarry }\end{array}$ \\
\hline & $\begin{array}{l}\text { Zona del puente } \\
\text { medieval } \\
\text { Medieval bridge zone }\end{array}$ & $\begin{array}{l}\text { Puente medieval } \\
\text { Medieval bridge }\end{array}$ & $\begin{array}{l}\text { Bancos escalonados, trinchera y cuñas } \\
\text { Staggered benches, trench and wedges marks }\end{array}$ \\
\hline $\begin{array}{l}\text { Cantera de Lieiro } \\
\text { Leiro quarry }\end{array}$ & & $\begin{array}{l}\text { Iglesia de Santa María de Lieiro } \\
\text { Church of Santa Maria de Lieiro }\end{array}$ & $\begin{array}{l}\text { Bancos de extracción } \\
\text { Extraction banks }\end{array}$ \\
\hline
\end{tabular}

Tabla 1. Signos observables en las diferentes áreas de extracción de piedra natural de San Ciprián y elementos del patrimonio asociado. Table 1. Observable marks in the different natural stone extraction areas in San Ciprián and elements of the associated heritage.

Figueiras. Es muy probable que se sitúen bajo las actuales edificaciones, aprovechando los huecos para las mismas.

Se puede seguir la evolución de las zonas de extracción de piedra para construcción a medida que el asentamiento fue avanzando geográfica y temporalmente, desde la zona de Porto de Riba, en épocas celta y romana, hasta los períodos más modernos de construcción de la localidad, durante el siglo XX.

La tecnología empleada para la extracción de dicha piedra fue evolucionando con el paso de los siglos. Se han localizado signos de explotación artesanal mediante cuñas en las zonas de extracción más antiguas (Porto de Riba, Figueiras y Lieiro), mientras que en las explotaciones más modernas o reutilizadas, se localizan signos de barrenos y un volumen de roca mayor, situándolas en un marco de explotación semiindustrial (Área de extracción E de Porto de Riba).

La extracción de piedra natural ha sido la actividad constante a lo largo de los siglos en San Ciprián, y ha producido importantes modificaciones en el paisaje, por lo que tiene una gran influencia visual integrada en la población, por lo que se propone su conservación como valor añadido a las construcciones de cara a futuras restauraciones de los mismos.

Aprovechando el interés turístico de la zona, su Patrimonio Monumental y la cercanía de las áreas de extracción a estos, se propone su puesta en valor mediante la creación de una ruta didáctica en la que se integre el patrimonio construido, la geología y las actividades histórico-extractivas, con el fin de acercar a la ciudadanía y turistas las relaciones existentes entre patrimonio construido y su entorno.

\section{Agradecimientos}

Los autores agradecen a la fototeca del Concello de Cervo, el acceso al material gráfico de su colección.

\section{Referencias}

Barro Quelle, M. 1989. San Ciprián. Parroquia de Lieiro. Serie Etnográfica limitada. Ediciós do Castro, Sada, 276 pp.

Fernández Rodríguez, C., González Gómez de Agüero, E. and Bejega García, V. 2008. Estudio del conchero del Castro de Punta Atalaia (San Cibrao, Lugo). Férvedes, 5. 43-52.

Fernández Suárez, J., Álvarez Areces, E. and Baltuille, J.M. 2016. Evolución histórico-geográfica de la extracción de piedra natural para la construcción civil y religiosa de San Ciprián (N de Lugo, Galicia). Geo-Temas, 16 (2), 375378.

Fernández Tomás, J., Monteserín López, V., Peinado Moreno, M. and Huerga Rodríguez, A. 1977. Hoja $n^{\circ} 3$ SAN CIPRIÁN. Mapa Geológico de España. E. 1:50.000. IGME, $2^{\text {a }}$ ser., $1^{\text {a }}$ ed., 14 pp. y 1 map.

Fort González, R. 2006. Utilización de la piedra natural en restauración. In. García del Cura, M.Á. and Cañaveras, J.C. (eds.), Utilización de rocas y minerales industriales. Seminarios de la Sociedad Española de Mineralogía, 2, Sociedad Española de Mineralogía, 155-182. 
García-Ruiz, J.L. 1986. La ciudad como recurso didáctico. I Congreso Español de Geología, 4, 505-525, Segovia.

Gutiérrez Claverol, M., Luque Cabal, C. and Pando González, L.A. 2012. Canteras históricas de Oviedo. Aportación al patrimonio arquitectónico. Hércules Astur de Ediciones, S.L., Oviedo, 256 pp.

Hernández Sampelayo, P. (1935): Criaderos de hierro de España. Tomo IV. Hierros de Galicia. Tomo III, fasc. $2^{\circ}$. Memorias del Instituto Geológico y Minero de España, 42. $769 \mathrm{pp}$.

Hose, T.A. 2000. 'Geoturismo' europeo. Interpretación geológica y promoción de la conservación geológica para turistas. In: Barretino, D., Wimbledon, W.A.P. and
Gallego, E. (eds.), Patrimonio Geológico: Conservación y Gestión. Instituto Tecnológico Geominero de España. Madrid. 137-161.

Riudavets, P. 1878. Carta Particular de la Ensenada y Puerto de San Ciprián en la costa de la provincia de Lugo H. 731 levantada en 1859 por el Teniente de Navío de la Armada Dn. Pedro Riudavets. Dirección de Hidrografía, Madrid.

Varas, $M^{a}$.J., Calvo, C. and Fort, R. 2010. Canteras Históricas: un recurso docente por explotar. Fundamental-XVI Simposio sobre Enseñanza de la Geología. Fundación Conjunto Paleontológico de Teruel, 269-282.

Xosé Ínsua, E. 2011. O porto de San Cibrao (Cervo) ao longo da historia. Ardentía, 6, 51-64.

Recibido: diciembre 2015

Revisado: febrero 2016

Aceptado: abril 2016

Publicado: junio 2017 